\title{
Heart Rate Variability in the Detection of Scares
}

\author{
Vinícius dos S. Oliveira \\ Department of Computing \\ Pontifical Catholic University of \\ Goiás, PUC-GO \\ Goiânia, Brazil
}

\author{
Edson D. F. de M. Santos \\ Department of Computing \\ Pontifical Catholic University of \\ Goiás, PUC-GO \\ Goiânia, Brazil
}

\author{
Talles M. de A. Barbosa \\ Department of Computing \\ Pontifical Catholic University of \\ Goiás, PUC-GO \\ Goiânia, Brazil
}

\begin{abstract}
To increase a user's satisfaction level when using an entertainment system it is extremely interesting that this system has the ability to obtain feedback from the user in real time. The emotional state of an individual is able to change his physiological systems behaviour. This paper proposes to analyze a physiological signal obtained through the photoplethysmography. The signal is collected using the multiparametric affective gamepad called Emopad. Furthermore, a heuristic is proposed to be implemented on the Emopad, to process and classify moments of scare an individual has while watching a scary video.
\end{abstract}

\section{General Terms}

Adaptive systems, Affective computing, Detection of Scares.

\section{Keywords}

Heart Rate Variability, Emopad, Scare Detection.

\section{INTRODUCTION}

The term Affective Computing has been cited for the first time at MIT media labs in 90's by Rosalind Picard [1]. Since then this recent field of computer science has expanded and grown inside research institutes. The objective of affective computing is to give computer systems the ability to identify, recognize, and express emotions. In addition, to comprehend and change the way they interact based on emotions.

Besides the use of Affective Computing in Human-Computer Interactions(HCI), it also expands to cover health related topics. Since most approaches to determine the emotional state of the user are through physiological signals, it is possible to infer beyond the emotional state to look for possible health problems using the signals. Point-of-care is the name given to this concept and has been explored in various systems, e.g., Smartphone, smart-watches, cars, health monitoring equipments and computers.

In the field of artificial intelligence, it has been shown that some intelligent systems work better if they take into account the dynamics of human emotions. For example, in a soccer video game the team changes its behaviour according to whether it is winning or losing. Another game that utilizes this concept is Nevermind. It is a game that uses the photoplethysmography (PPG) sensor to detect the heart rate variability of the player, to determine if he is in a state of stress. After determining the player's stress level, it changes the games difficulty, so if the player wants to reduce the difficulty he has to learn to control his stress.

The first approach for automatic detection of scares in the Emopad project was by using the GSR as input data for analysis [2]. That was the work that gave rise to this Emopad project. It was possible to detect scares using the GSR; however, there were too many false positives.

\section{MATERIALS AND METHODS}

\subsection{Photoplethysmography Acquisition}

Photoplethysmography (PPG) is a process where a user's blood volume pulse is measured. The sensor used to obtain the PPG from the players was developed by Pulse Sensor [3]. This sensor contains an embedded circuit that filters the signal and takes the first derivative of the filtered signal.

In order to represent the signal with enough details to detect a cardiac beat and be energy-efficient while processing the data a sampling rate of $20 \mathrm{~Hz}$ was ideal in meeting both requirements.

\subsection{Dataset}

In the study relating the GSR for use in the detection of scares a database was generated containing physiological responses of players. These responses were obtained using the Emopad while the respective players played a game of terror called "Death Unknown". While playing the game their physiological signals, and facial expressions were being collected and stored in a computer. With each physiological signal there would be a corresponding video recording that was used to observe when there was a moment of scare. CollectPhys is a software developed in [2] and was used to plot and store the data in the computer.

Each person has a way of playing a game resulting in different paths and events. The resulting timelines will differ from person to person. Therefore, for this work a new database was constructed in a more controlled manner that stimulates scares. A video was chosen for the stimuli as it would not change its scenes or sequences for each person. Thus, there is greater control over which stimuli is exhibited and expected.

To study the patterns generated in the physiological signals arising from emotions, it is necessary to keep the user at rest for at least two minutes. This will stabilize the physiological system before we stimulate the desired emotion [4]. Taking deep breaths is one of the best ways to auto-stabilize the physiological system. These are the first two steps in the video respectively. In the third step, the short film "Lights Out" is played to stimulate scares. After the short film the next step is a short rest of two minutes to return the person to a state of stability. The step with a mathematic problem serves to induce mental stress, this is by counting in descending order from a hundred.

The database constructed for the development of the heuristic, contains samples from 13 participants, 5 male individuals and 8 female individuals. In the database four samples were discarded due to a failure in transmission to the PC. 


\subsection{Automatic Detection of Heart Rate Variability (HRV)}

Initially the algorithm provided by Pulse Sensor was used for the PPG. This algorithm performs well and recognizes heartbeats when the signal from the PPG is clean. While collecting the samples of PPG some would have noises due to the participant's hand movements in relation to the sensors. This caused the Pulse Sensor algorithm to sometimes not be able to recognize heartbeats. The a-Wave detection algorithm [10] proved much better in detecting the cardiac beats when the signal would suffer from this type of noise as seen in Figure 1. There is a difference in the results from both algorithms during a period of noise. The HRV signal is composed by calculating the intervals of heart beats, called R$\mathrm{R}$ or N-N intervals. So, the HRV signal represents the periods of consecutively heartbeats.
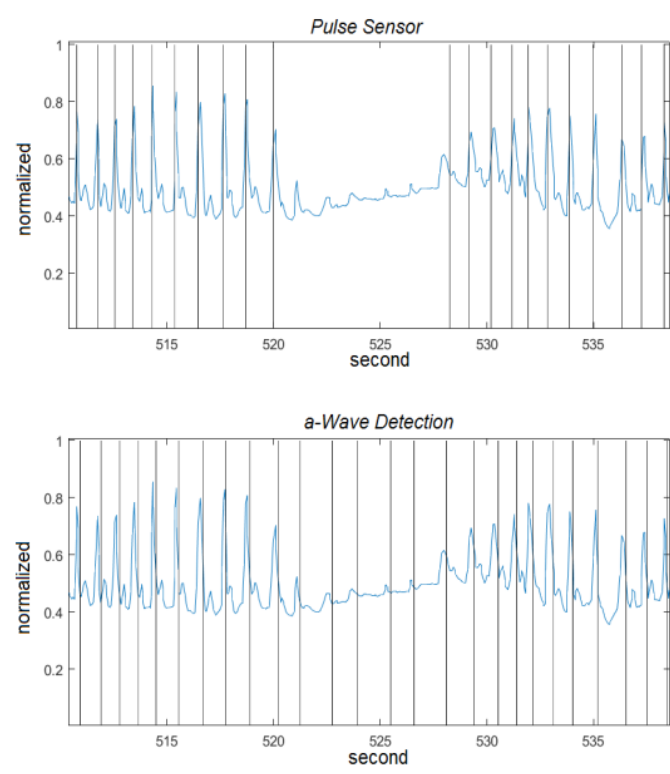

Figure 1: Comparison between Pulse Sensor's Algorithm and a-Wave Detection Algorithm. The vertical lines represents the detections of heartbeats. The blue line is the signal obtained by PPG sensor.

\subsection{Analysis of SDNN and pNN50 in the detection of a scare}

The standard deviation of the HRV (SDNN) and the percentage of $\mathrm{N}-\mathrm{N}$ intervals that differentiate by more than $50 \mathrm{~ms}$ (pNN50), are the principal characteristics for the detection of stress [4]. Based on this, an analysis was proposed to detect scares since in a moment of scare there is a brief interval of stress. A scare causes the body to have an involuntary reaction signaling the brain that there might be danger, heart rate increases, the blood vessels contract to give priority to the leg muscles, the body prepares to either sprint from danger or fight.

The SDNN serves as an indicator for stress levels. When someone is in a state of stress the systems that control the heart rate goes into conflict and the heart rate variability decreases. The standard deviation is calculated based on
Equation 1 and pNN50 is based on the pseudo-code illustrated on Figure 2.

Both the SDNN and pNN50 were calculated using short window sizes of $10 \mathrm{~s}$ and shifting size of $1 \mathrm{~s}$. In another study it was demonstrated that using window-sizes similar to these configurations, the results can be close or similar to medical grade equipments [4] which on average uses windows of 5 minutes. Small window sizes were used to save processing and have faster responses in the behaviour of the HRV.

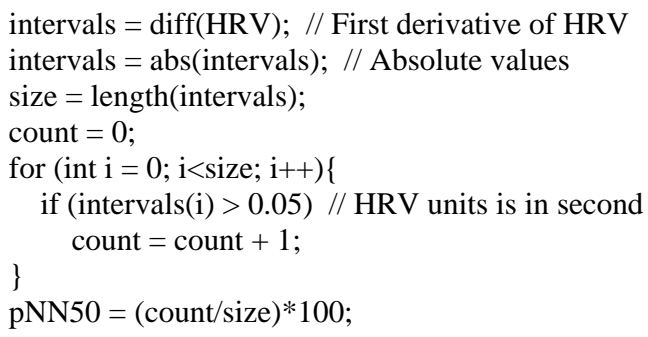

Figure 2: Calculation of pNN50

$$
\begin{aligned}
& =\sqrt{\frac{1}{n-1} \sum_{i=1}^{n}\left(x_{i}-\bar{x}\right)^{2}}
\end{aligned}
$$

The SDNN and pNN50 was not a good indicator for scares. In some cases, it showed significant change in moments of scare. However, these behaviors were very rare, so we unclassified the possibility of recognizing scares using the SDNN and pNN50.

\subsection{Proposed Heuristic}

The behavior that most stood out during the scares was the increase of the heart. The heuristic proposed in this paper, evaluates these variations of the heart rate to indicate a moment where a scare has occurred. The heuristic activities are described in Figure 3.

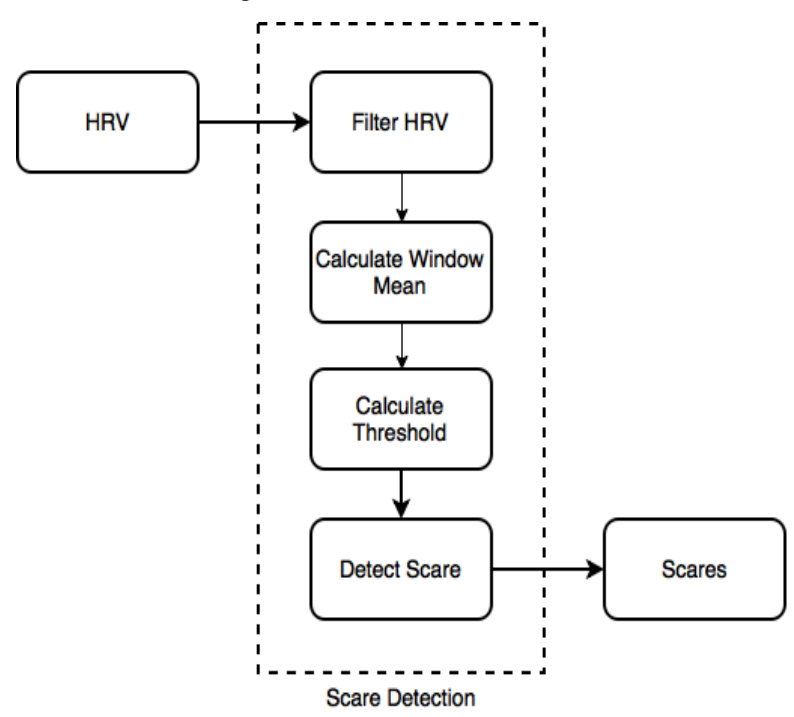

Figure 3: Activities of Heuristic 


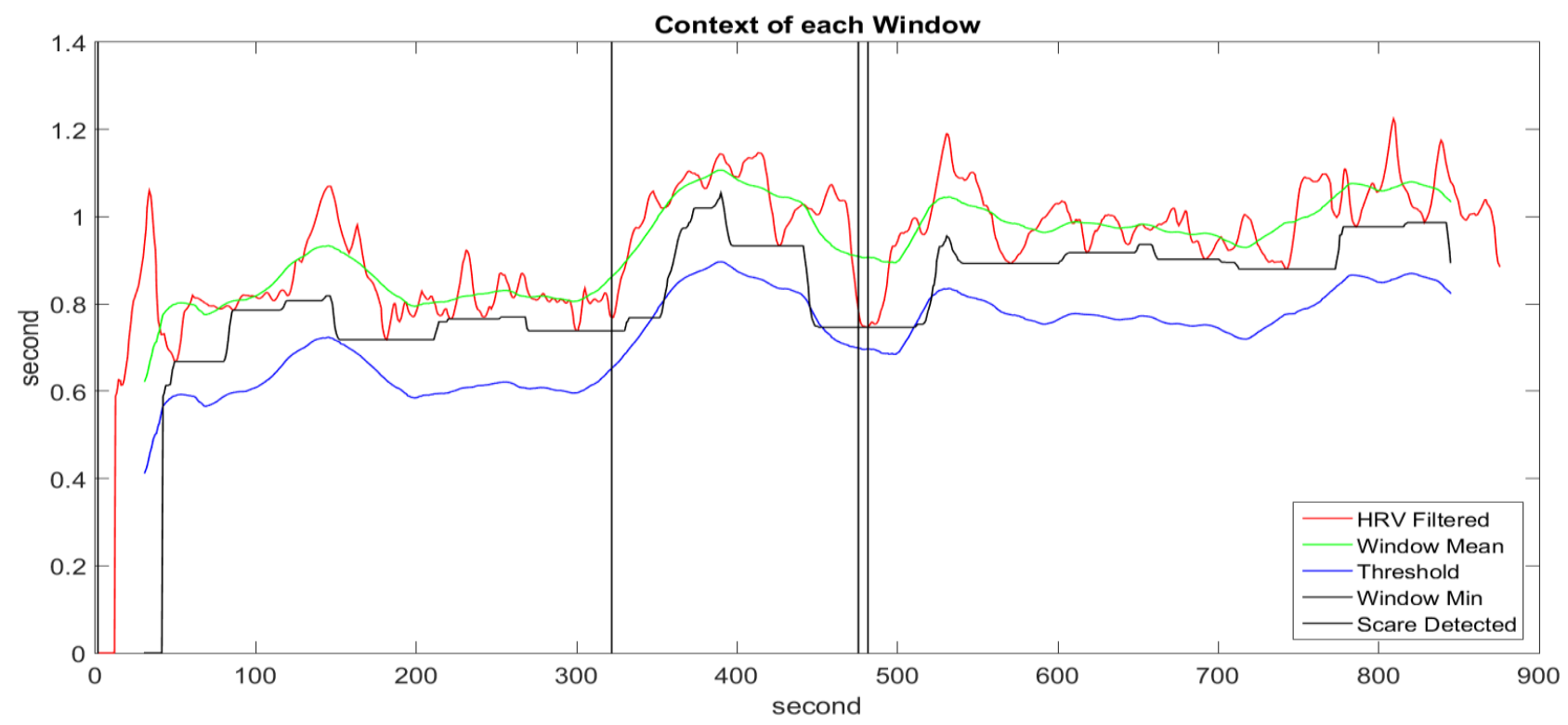

Figure 4: Context of each window and scare detections

The window size is equal to $60 \mathrm{~s}$ and the shifting size equal to 1s. This configuration was based on the heart response time to sympathetic stimulation, varying from 5 to almost 30 seconds. Since there is a need to include the recovery period after the scare in the window it is necessary to add 30 s, adding up to $60 \mathrm{~s}$.

Inside of each window the arithmetic mean is calculated using the previously filtered HRV signal. Then a sum is made on this average value to calculate a limit, which serves as a threshold to define when there is a scare. The minimum is also found within each window. If this minimum is lower than the threshold, that moment is considered a scare. The decision rules described are represented by the automaton in Figure 5.

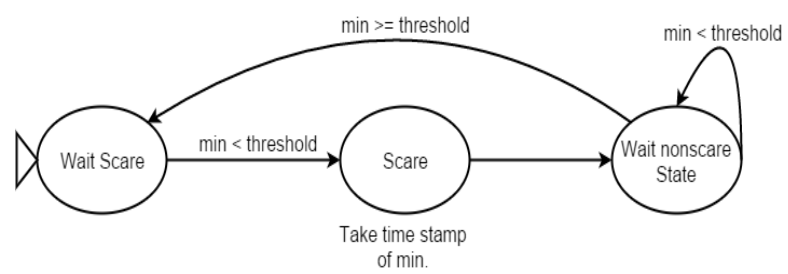

Figure 5: Rules of Heuristic

The context of each window during the processing of a sample is shown in Figure 4. The red line in Figure 4, represents the filtered HRV. The green line represents the mean of each window. The blue line represents the threshold calculated by the sum of the mean with a floating number. The black line represents the minimum found in each window. Note that when the minimum is lower than the threshold, a scare is detected the moment the minimum is found.

\subsection{Test and Validation}

First of all, the proposed heuristic was evaluated using the database obtained using the video as its stimuli. The results of this analysis can be seen on Table 1. Then the same heuristic was evaluated using the database from Bachinni's work [2]. The results of this analysis is shown on Table 2. The evaluation of the heuristic was based on [6]. The evaluation rates were calculated using equations 2 and 3 .
Table 1. Results of Heuristic over video database

\begin{tabular}{|c|c|c|c|}
\cline { 3 - 4 } \multicolumn{2}{c|}{} & \multicolumn{2}{c|}{ Actual } \\
\cline { 3 - 4 } \multicolumn{2}{c|}{} & Scare & Non-Scare \\
\hline \multirow{2}{*}{ Predicted } & Scare & 6 & 6 \\
\cline { 2 - 4 } & Non-Scare & 7 & 4775 \\
\hline
\end{tabular}

Table 2. Results of Heuristic over video-game database

\begin{tabular}{|c|c|c|c|}
\cline { 3 - 4 } \multicolumn{2}{c|}{} & \multicolumn{2}{c|}{ Actual } \\
\cline { 3 - 4 } \multicolumn{2}{c|}{} & Scare & Non-Scare \\
\hline \multirow{2}{*}{ Predicted } & Scare & 12 & 17 \\
\cline { 2 - 4 } & Non-Scare & 96 & 21261 \\
\hline
\end{tabular}

True Scare Detection (TSD) is the rate of positive accusations of a scare, based on equation 2 . The terms used in the equations corresponds to all the windows which falls in the classifications of: True Positive, True Negative, False Positive and False Negative. True Positive is classified when a window accuses a scare in which the participant had a real scare (seen on Webcam Recording). False Positive is classified when a window accuses a scare in which the participant did not have a real scare. The remaining classifications follows the same logic.

$T S D$

$$
=\frac{\text { True Positive }}{(\text { True Positive }+ \text { False Negative) }}
$$

True Non-Scare Detection (TSND) is the rate of negative accusations of a scare, based on equation 3 .

TSND
$=\frac{\text { True Negative }}{\text { (True Negative }+ \text { False Positive })}$ 

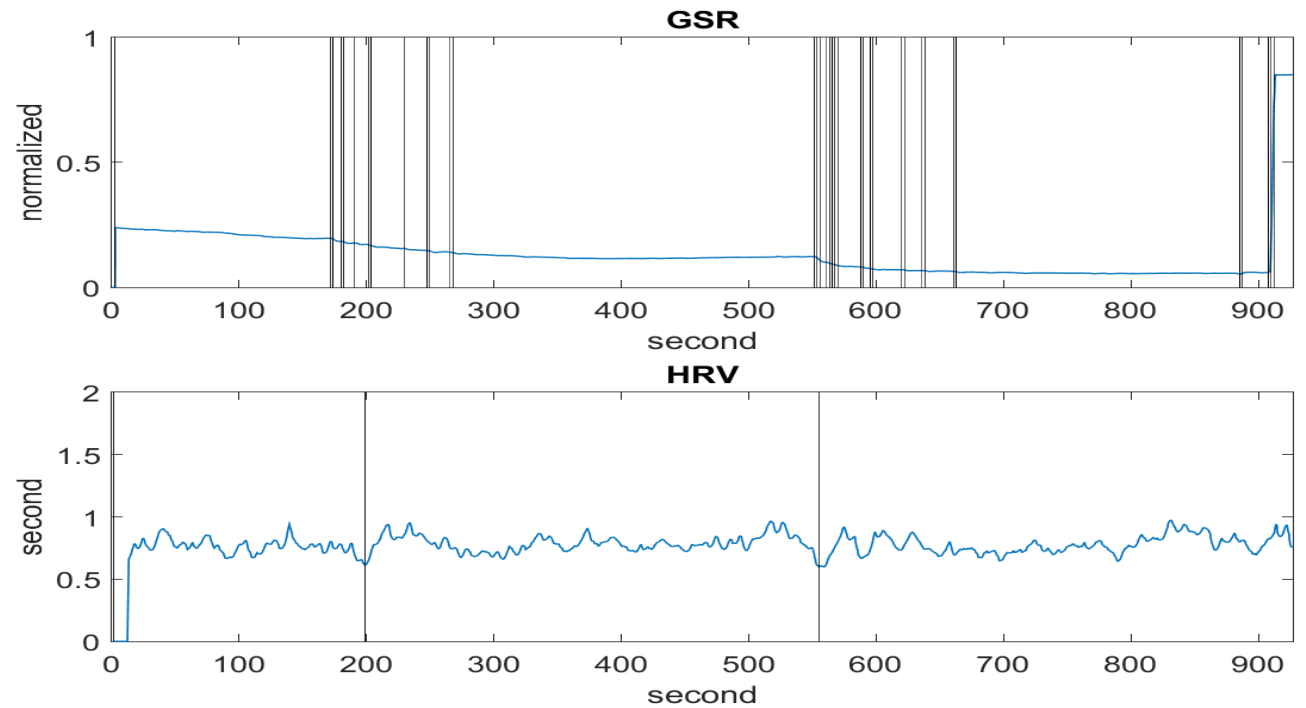

Figure 6: Results using GSR and HRV to detect scares.

The TSD rate of the proposed heuristic using the database of the video was of $50 \%$, compared to a rate of $41,38 \%$ while using the video-game database. The TSD value achieved with the use of GSR on previous work was of $100 \%$. The TNSD rate while using the video database had a rate of $99,85 \%$, compared to a $99,55 \%$ when using the video-game database. In previous work when using the GSR the TNSD rate was 95,5\%. Therefore, the proposed heuristic in this paper has a lower rate for TSD; however, the number of false positives(vertical lines) found has been substantially lower as seen in Figure 6.

\section{CONCLUSION}

Even though the TSD rate while using the HRV for scare detection was lower than the GSR, the amount of false positive generated with the proposed heuristic in this work was significantly less, as showed in Figure 6.

During analysis of the proposed heuristic it was noted that most of the false accusations of a scare were in fact due to anxiety. For instance, the moment a player would open a door he would expect something to scare him, thus creating a certain anxiety. The moments of anxiety a person has can be useful information for a game designer.

For future works there can be an approach to work on analyzing the proposed heuristic in this paper to recognize those moments of anxiety that can cause false positives. Another one is to concentrate on the data fusion between the results of GSR and HRV to further improve the pattern recognition accuracy of a scare. Many improvements can be made to the Emopad, regarding its autonomy, redistribution of the sensors to allocate them in better holding places, as well as redundancies of the sensors. The re-positioning of some sensors is important due to the fact that in some cases the sensors would not be in contact with the skin for a brief moment.

\section{ACKNOWLEDGMENTS}

This study was supported by FAPEG (in portuguese, Fundação de Amparo à Pesquisa de Goiás) at Pontifical Catholic University of Goiás. We are grateful to all those involved in this project, especially Erlan Lopes for their valuable contributions.

\section{REFERENCES}

[1] R. Picard, "Affective computing", MIT Press. Boston, 1997.

[2] Bacchini, Pedro HF, et al. "Developing an affective Point-of-Care technology."Computational Intelligence in Healthcare and e-health (CICARE), 2014 IEEE Symposium on. IEEE, 2014.

[3] Joel Murphy and Yury Gitman. (2015, Dec. 03). Pulse Sensor. [Online]. Available: pulsesensor.com

[4] MEDICORE, "Heart Rate Variability Analysis System", Website: http://medicore.com/download/HRV_clinical_manual_ver3.0.pdf [16 March 2015]

[5] K. Schaaff and M. T. P. Adam, "Measuring Emotional Arousal for Online Applications: Evaluation of UltraShort Term Heart Rate Variability Measures" Proceedings of Humaine Association Conference on Affective Computing and Intelligent Interaction, 2013.

[6] A. S. Sierra, C. S. Ávila, J. G. Casanova and G. B. del Pozo, "A Stress-Detection Based on Physiological Signals and Fuzzy Logic", IEEE Transactions on Industrial Electronics, v. 58, n. 10, october 2011.

[7] Loggia, Marco L., Mylène Juneau, and M. Catherine Bushnell. "Autonomic responses to heat pain: Heart rate, skin conductance, and their relation to verbal ratings and stimulus intensity." PAIN® 152.3 (2011): 592-598.

[8] Hey, Stefan, et al. "Continuous noninvasive pulse transit time measurement for psycho-physiological stress monitoring." eHealth, Telemedicine, and Social Medicine, 2009. eTELEMED'09. International Conference on. IEEE, 2009.

[9] Johnston, William S. Development of a Signal Processing Library for Extraction of $\mathrm{SpO}$, HR, HRV, and RR from Photoplethysmographic Waveforms. Diss. WORCESTER POLYTECHNIC INSTITUTE, 2006.

[10] Elgendi, Mohamed, Mirjam Jonkman, and Friso DeBoer. "Heart Rate Variability and the Acceleration Plethysmogram signals measured at rest."Biomedical 
Engineering Systems and Technologies. Springer Berlin Heidelberg, 2011. 266-277.

[11] Tivatansakul, S., and M. Ohkura. "Improvement of emotional healthcare system with stress detection from ECG signal." Engineering in Medicine and Biology Society (EMBC), 2015 37th Annual International Conference of the IEEE. IEEE, 2015.

[12] Heart Rate Variability. "Standards of measurement, physiological interpretation, and clinical use. Task Force of the European Society of Cardiology and the North American Society of Pacing and Electrophysiology."Circulation 93 (1996): 1043-1065.
[13] J. Hsu, "Game Controller Senses Players' Excitement Levels", online: http://spectrum.ieee.org/techtalk/consumer-electronics/gaming/game-controllersenses-players-excitement-levels, April, 2014.

[14] Game Jolt, "Death Unknown". Online http://gamejolt.com/games/other/death-unknown-v-12/16329/'

[15] P. H. F. Bacchini, J. O. Ferreira and T. M. G. de A. Barbosa, "Emopad: An Affective Gamepad", International Journal of Computer Applications, vol. 102, no. 15 , pp.6-12, Sept. 2014. doi: 10.5120/17889-8885 